\title{
The Influence of Several Biological Parameters on the Management of Patients with Colon Cancer Undergoing Elective Surgery
}

\author{
Cătălin Vlăduț Ionuț Feierr ${ }^{1,2, *}$, Andra Olariu ${ }^{3, *}$ and Sorin Olariu ${ }^{\text {I,2 }}$ \\ ${ }^{\text {I }}$ First Surgery Discipline, Department X, "Victor Babes” University of Medicine and Pharmacy, \\ 30004I Timisoara, Romania; srnolariu@yahoo.com \\ ${ }^{2}$ First Surgery Clinic, "Pius Branzeu" Clinical Emergency Hospital, 300736 Timisoara, Romania \\ 3 Faculty of Medicine, "Victor Babes" University of Medicine and Pharmacy, 30004ITimisoara, Romania \\ * Correspondence: catalinfeierı@@gmail.com (C.V.I.F.); andraaolariu@hotmail.com (A.O.); \\ Tel.: +40-748-027590 (C.V.I.F.)
}

Submitted: 23 December 2021; Accepted: 2 February 2022; Published: 16 February 2022

\begin{abstract}
I) Aim of the study: Our study aims to analyze the influence of biological parameters such as hemoglobin, albumin, total protein, on the management and duration of treatment of colon cancer patients who have undergone elective surgery. (2) Material and methods: Data of 147 patients from the Surgery Clinic I of the County Clinical Hospital of Timisoara who underwent an elective surgery for colon cancer treatment were statistically analyzed using statistical tests such as Anova or Chi square with a $p<0.05$ considered to be statistically significant. (3) Results: Differences between preoperative and postoperative parameters were correlated with the duration of surgery (hemoglobin, $p=0.014$ ). The average length of hospital stay was correlated with the difference between the preoperative and postoperative level of hemoglobin $(p=0.043)$. There was a direct correlation between the length of postoperative hospital stay and the difference between the plasmatic levels of albumin $(p=0.033)$. There were differences in the average Charlson index value of patients who required albumin, with no statistical significance. (4) Conclusions: The difference between preoperative and postoperative levels of biological parameters (hemoglobin, albumin, total protein) has an influence on the management of patients with colon cancer undergoing elective surgery, affecting the number of days the patients spend in the hospital after the surgical procedure, as well as the total number of days spent in the hospital (from day one to discharge). The greater the variation of those differences, the longer recovery period needed, showing that an early correction of those parameters can lead to a shorter hospital stay, a faster postoperative recovery and a shorter postoperative hospital stay.
\end{abstract}

Keywords: colon cancer; elective surgery; albumin; total protein; hemoglobin

How to cite: Feier, C.V.I.; Olariu, A.; Olariu, S. The Influence of Several Biological Parameters on the Management of Patients with Colon Cancer Undergoing Elective Surgery. Timisoara Med. 2022, 2022(I), 2; doi:10.35995/tmj20220102. 


\section{Introduction}

Globally, colon cancer accounts for Io\% of all malignancies. This condition is the third most common form of cancer in men and the second most common in women. Colon cancer is responsible for the deaths of 600,000 people each year, making it the fourth-highest cancer mortality cause [I,2]. In Europe, 5 -year survival varies from $30.9 \%$ to $60 \%$ in female patients, and from $28.5 \%$ to $57 \%$ in male patients [3].

The COVID-Is pandemic had a major influence on the management and treatment of colon cancer. The SARS-CoV-2 virus appeared in Wuhan and then spread globally, becoming a public health emergency. Since all health systems around the world have been overwhelmed by the fast spread and the high numbers of patients infected with the new coronavirus, restrictions have been imposed. People around the world have been advised to reduce contact with others, and to visit hospitals only in case of a severe symptomatology.

The success of elective surgery for the treatment of colon cancer is influenced by several key biological parameters such as the levels of hemoglobin, albumin and total plasma proteins, each one playing an important role in the patient's postoperative recovery. In addition to these parameters, the patients' comorbidities have an important role in the postoperative recovery. This study presents the influence of these parameters on the treatment and management of patients undergoing elective surgery for the treatment of colon cancer.

\section{Materials and Methods}

This study was carried out in the ist Surgery Clinic of the Clinical Emergency County Hospital, Timișoara, Romania, analyzing data from three distinct periods of time: I March 2016-30 September 2017, I March 2018-30 September 2019, respectively, I March 2020-30 September 2021. Only the patients who underwent elective surgery for the treatment of colon cancer were taken into consideration.

The inclusion criteria were the following: patients who underwent elective surgery for the treatment of colon cancer, within the studied period; additionally, during the pandemic period, more inclusion criteria were added: the absence of infection or recent history of infection with SARS-CoV-2 virus, absence of any specific symptoms of infection for at least 7 days prior to presentation, a negative PCR test at the admission to the hospital. The exclusion criteria were included the need for an emergency surgery and, during the pandemic, by the presence or recent history of the new coronavirus infection, the presence of any symptoms characteristic of this infection at presentation day or 7 days prior to hospitalization, as well as a positive result for a PCR test.

The data collection was conducted with the approval of the Ethics Commission of the Hospital. Regarding the demographic parameters, the age, the gender, the rural or urban origin were analyzed. Biological parameters such as hemoglobin level before and after the surgery were studied, with a $\mathrm{dHb}$ variable representing the value of the difference between the two moments. The same method was applied for the variable dAlb (representing the difference between the albumin levels before and after surgery), and the variable dProt (representing the difference between the total plasmatic protein levels). $\mathrm{Na}$ and $\mathrm{K}$ levels were analyzed, as well as the duration of surgery, the duration of postoperative hospitalization and the total duration of hospitalization. The need for postoperative erythrocyte concentrate transfusions as well as patient comorbidities were also taken into consideration. Comorbidities were assessed using the Charlson Index.

\section{Statistical Methods}

All data were analyzed retrospectively. The categorical variables were compared using Chi square tests and a $p<0.05$ was declared statistically significant. For the numerical variables, the parameters of the central tendency 
and those of dispersion were used, as well as unpaired t tests or Anova tests. Where appropriate, non-parametric Mann-Whitney tests were applied. All data processing was performed using the SPSS statistical analysis software program.

\section{Results}

The demographic aspects of the patients in the study are presented in Table I.

Table I. Demographical aspects of the patients.

\begin{tabular}{ccc}
\hline & & $\mathbf{N}(\%)$ \\
\hline Gender & Male & $78(53 \%)$ \\
& Female & $69(47 \%)$ \\
\hline \multirow{2}{*}{ Environment } & Urban & I04 $(70.74 \%)$ \\
& Rural & $43(29.25 \%)$ \\
\hline
\end{tabular}

Between 2020 and 202I, data from 29 patients were taken into consideration, representing $19.72 \%$ of the total number of cases, compared to 68 patients $(46.25 \%)$ in $2018-2019$ and 50 patients $(34.01 \%)$ in 2016-2017.

The age of the patients varied from 37 to 87 years, with an average of 65.75 years, a standard deviation of 9.98 years, and a median of 66 years.

Initially we have analyzed the plasma levels of sodium and kalium electrolytes. The data are presented in Table 2.

Table 2. Variation of the plasmatic levels of $\mathrm{Na}$ and $\mathrm{K}$.

\begin{tabular}{ccccc}
\hline & & 2016-20I7 & 2018-2019 & 2020-202I \\
\hline \multirow{2}{*}{$\mathbf{N a ~ ( m m o l / L ) ~}$} & Average & I39.69 & I40.12 & 138.52 \\
& Standard Deviation & 3.37 & 4.05 & 5.13 \\
& Median & I40 & I4I & I 40 \\
\hline \multirow{2}{*}{$\mathbf{K}(\mathbf{m m o l} / \mathbf{L})$} & Average & 4.30 & 4.43 & 4.21 \\
& Standard Deviation & 0.52 & 0.53 & 0.69 \\
& Median & 4.3 & 4.5 & 4.2 \\
\hline
\end{tabular}

The following step of our analysis was to compare the differences between the preoperative and postoperative levels of hemoglobin $(\mathrm{dHb})$, albumin (dAlb), and plasma proteins (dProt). The results are presented in Table 3 .

A statistical analysis of the correlation between plasma protein difference and plasma albumin showed a $p<0.001$ over the three periods of time. In the period 2016-2017, a $p=0.011$ was obtained, in the second period $p=0.018$, and in the pandemic period $p=0.002$.

It should be mentioned that in the period 2016-2017 the correlation between albumin (dAlb) and hemoglobin $(\mathrm{dHb})$ generated $\mathrm{a} p=0.039$. 
Table 3. Differences of biological parameters before and after the surgery.

\begin{tabular}{ccccc}
\hline & & 2016-2017 & 2018-2019 & 2020-202I \\
\hline \multirow{2}{*}{$\mathbf{d H b}(\mathbf{g} / \mathbf{d L})$} & Average & $0.16^{* \#}$ & $\mathrm{I}^{*} \mathrm{I}^{*}$ & $\mathrm{I} .3^{* \#}$ \\
& Standard Deviation & 2.09 & 2.4 & 2.26 \\
& Median & 0.4 & $\mathrm{I} .4$ & $\mathrm{I} .3$ \\
\hline \multirow{2}{*}{$\mathbf{d A l b}(\mathbf{g} / \mathbf{d L})$} & Average & 0.785 & 0.77 & 0.49 \\
& Standard Deviation & 0.59 & 0.6 & 0.56 \\
& Median & 0.7 & 0.95 & 0.3 \\
\hline \multirow{2}{*}{$\mathbf{d P r o t}(\mathbf{g} / \mathbf{d L})$} & Average & I.25 & I.05 & 0.86 \\
& Standard Deviation & 0.74 & I.06 & 0.5 \\
& Median & 1.45 & $\mathrm{I}$ & 0.85 \\
\hline
\end{tabular}

${ }^{*} p=0.046$ between all 3 periods of time; ${ }^{\#} p=0.042$ between $2016-2017$ and $2020-2021$.

Regarding the transfusions received by patients, they were present in $22 \%$ of cases in the first period, $10.29 \%$ of cases in the period $2018-2019$ and $13.79 \%$ of cases during the pandemic.

The average age of patients who needed postoperative albumin was 70.25 years, compared to 63.97 of those who did not need it.

The mean duration of hospitalization was 18.72 days in patients who had a decrease in hemoglobin of at least $0.5 \mathrm{~g} / \mathrm{dL}$ postoperatively and did not receive blood transfusions, while in those who received transfusions the mean duration of hospitalization was 16.32 days. Regarding the study of the average duration of hospitalization correlated with an increase in $\mathrm{dHb}, \mathrm{a} p=0.043$ was obtained.

The correlation between the difference in hemoglobin level before and after surgery with the duration of the operation was studied and a $p=0.014$ was obtained. When studying a possible correlation between the magnitude of $\mathrm{dAlb}$ and the duration of the days of hospitalization after the intervention, $\mathrm{a} p=0.033$ was obtained. Regarding the level of albumin, those who showed a decrease of at least $0.5 \mathrm{~g} / \mathrm{dL}$ and did not receive albumin had an average hospital stay of $20.8 \mathrm{I}$ days compared to an average duration of $\mathrm{IS}$ days of those who received postoperative albumin.

In studying the variation of the Charlson index in association with dAlb, patients who received albumin had an average Charlson index of 5.25 versus 3.97 of the ones that did not require postoperative albumin.

\section{Discussion}

With the onset of the COVID-I9 pandemic, the number of patients presenting to hospital for elective surgical treatment of colon cancer has decreased compared to previous periods. During the pandemic, there were 29 patients, compared to 68 in 20I8-2019 and 50 in 2016-2017. This is largely due to pandemic restrictions such as visiting medical centers only if the patient had severe symptoms, patients' fear of contacting the new coronavirus, and the hospital's focus on treating patients with COVID-19. During the pandemic, this decrease in the number of cases occurred in countries such as Italy where there were $39 \%$ fewer patients that underwent an elective surgery for colon cancer treatment [4].

In this study, various biological parameters of the patients were analyzed. One of these was the hemoglobin value. The magnitude of the difference between preoperative hemoglobin and postoperative hemoglobin was 
studied. This difference was the smallest in the period 2016-2017 with an average of $0.16 \mathrm{~g} / \mathrm{dL}$, and the highest I.4I $\mathrm{g} / \mathrm{dL}$ in the period 2018-2019. During the pandemic, the average difference was I.3 $\mathrm{g} / \mathrm{dL}$. Following the Anova test, we obtained a $p=0.046$, showing that there are significant differences over the three periods of the study in terms of $\mathrm{dHb}$ levels. After applying the $\mathrm{T}$ test for the period 2016-2017, compared to 2020-202I, it generated a $p=0.042$, resulting in statistically significant differences between the two periods, showing that patients had a lower level of postoperative hemoglobin in the pandemic period, they presented to the hospital in a more advanced stage of the disease, as well as with a more severe symptomatology. This is also confirmed by the study of Cui et al. from 202I, which states that there were $20 \%$ more patients with severe symptoms during the pandemic [5].

It is worth mentioning that the percentage of patients who received blood transfusions increased from I0.29\% during 2018-2019 to $13.79 \%$ during the pandemic. An increase in mean age was observed in parallel with the need for transfusions. This is due to the need of the older patients to be helped to recover, as the physiological processes slow down with age. This is documented in other articles that show older patients have a lower hemoglobin level, and therefore a lower regenerative capacity [6].

The length of hospitalization was influenced by $\mathrm{dHb}$. during the three periods. For patients with a postoperative decrease greater than $0.5 \mathrm{~g} / \mathrm{dL}$ in hemoglobin who did not receive blood transfusions, there was an average of 18.72 days of hospitalization, whereas in patients who received postoperative blood transfusions, the average number of days of hospitalization was 16.32 days. This is due to the body's regeneration capacity, which is influenced by higher levels of postoperative hemoglobin. Following the correlation analysis, a $p=$ 0.043 was obtained, showing that there is a correlation between the increase in $\mathrm{dHb}$ and the average length of hospitalization. As the patient had lower postoperative hemoglobin levels and involved a higher $\mathrm{dHb}$, it had a longer recovery period and, as a result, a prolonged hospitalization.

Moreover, the connection between the $\mathrm{dHb}$ variation and the duration of the intervention was analyzed, and $\mathrm{a} p=0.0 \mathrm{I} 4$ was obtained, showing that there is a correlation between the two variables. Thus, a prolonged operation time implies a higher degree of difficulty of the surgical act, and a prolonged exposure to the hemorrhagic risk.

Regarding the parameters such as albumin and plasma proteins, the correlation between these two was studied, and a $p<0.00$ r resulted in the correlation between albumin and total proteins. It should be noted that, in the period 2016-2017, a $p=0.039$ was obtained, which shows a correlation between $\mathrm{dAlb}$ and $\mathrm{dHb}$, so in that period the patients whose hemoglobin value decreased, had in parallel a decrease in postoperative albumin value.

Patients who required postoperative albumin had a mean age of 70.25 years, compared to 63.97 years for those not needing albumin therapy. This confirms the inability of the elderly body to cope with surgery if not helped as seen in other studies such as the one from Ristescu et al. $[7,8]$.

The average length of hospital stay was influenced by dAlb. Thus, patients who had a decrease of more than $0.5 \mathrm{~g} / \mathrm{dL}$ and did not receive albumin had an average hospital stay of 20.8I days, compared to Is days for patients who received albumin. This is due to the need for the body to have at its disposal albumin and plasma protein for faster postoperative recovery, for the integrity of the anastomosis performed after surgery, and to avoid postoperative complications such as anastomotic leak. Finally, the more balanced a patient was, the quicker he left the hospital environment. 
The average duration of postoperative days generated a significant value of $p=0.033$ in terms of correlation with the magnitude of albumin. Thus, a correlation was observed between the two variables, showing that with the increase in the dAlb, the number of days spent in the hospital after the surgery increases. This aspect has also been found in the research articles such as the one from Haskins et al., where a $p<0$.oor has been described [9].

Regarding the Charlson Comorbidity Index and its association with dAlb, it was observed that patients who needed postoperative albumin had an average Charlson Index of 5.25, and those who did not need it had an average index of 3.97. This association shows that patients with comorbidities have a higher degree of difficulty in postoperative recovery, requiring additional monitoring and albumin to avoid postoperative complications [Iо,II].

\section{Conclusions}

Following this study, differences were observed statistically over the three studied periods in terms of hemoglobin difference level; moreover, in the period 2016-2017, a correlation between $\mathrm{dAlb}$ and $\mathrm{dHb}$ was observed, as well as a correlation between the average duration of hospitalization and $\mathrm{dHb}$. Furthermore, the duration of the surgery was correlated with $\mathrm{dHb}$, showing that with the increase in the complexity of the cases, there was an increase in the duration of the surgery, as well as an increase in $\mathrm{dHb}$. Patients' comorbidities also had an influence on dAlb, so that the higher the Charlson Index was, the greater was the need for albumin. The greater the variation of those differences was ( $\mathrm{dHb}, \mathrm{dAlb}, \mathrm{dProt})$, the longer recovery period was needed, showing that an early correction of those parameters can lead to a shorter hospital stay, a faster postoperative recovery and shorter period of time until discharge.

Author Contributions: Conceptualization, C.V.I.F., A.O. and S.O.; methodology, C.V.I.F., A.O., S.O.; software, C.V.I.F.; validation, C.V.I.F., A.O. and S.O.; formal analysis, S.O.; investigation, C.V.I.F., A.O. and SO; resources, C.V.I.F., A.O. and S.O.; data curation, C.V.I.F..; writing-original draft preparation, C.V.I.F., SO; writing-review and editing, C.V.I.F., A.O. and S.O.; visualization, C.V.I.F., A.O. and S.O.; supervision, S.O.; project administration, C.V.I.F.

Funding: This research received no external funding.

Conflicts of Interest: The authors declare no conflict of interest.

\section{References}

I. Arnold, M.; Sierra, M.S.; Laversanne, M.; Soerjomataram, I.; Jemal, A.; Bray, F. Global patterns and trends in colorectal cancer incidence and mortality. Gut 2017, 66, 683-691. [CrossRef] [PubMed]

2. Allemani, C.; Weir, H.K.; Carreira, H.; Harewood, R.; Spika, D.; Wang, X.S.; Bannon, F.; Ahn, J.V.; Johnson, C.J.; Bonaventure, A.; et al. Global surveillance of cancer survival 1995-2009: Analysis of individual data for 25,676,887 patients from 279 population-based registries in 67 countries (CONCORD-2). Lancet 2015, 385, 977-IOIO. [CrossRef]

3. Torre, L.A.; Siegel, R.L.; Ward, E.M.; Jemal, A. Global cancer incidence and mortality rates and trends-An update. Cancer Epidemiol. Biomark. Prev. 2016, 25, 16-27. [CrossRef] [PubMed]

4. Choi, J.; Park, I.; Lee, H.; Cho, E.; Kim, Y.; Kim, C.; Yoon, Y.; Lim, S.-B.; Yu, C.; Kim, J. Impact of the COVID-i9 Pandemic on Surgical Treatment Patterns for Colorectal Cancer in a Tertiary Medical Facility in Korea. Cancers 202I, $I_{3}$, 222I. [CrossRef] [PubMed]

5. Cui, J.; Li, Z.; An, Q.; Xiao, G. Impact of the COVID-19 Pandemic on Elective Surgery for Colorectal Cancer. J. Gastrointest. Cancer 202I. [CrossRef] [PubMed]

6. Ristescu, I.; Pintilie, G.; Filip, D.; Jitca, M.; Fecheta, R.; Florescu, I.; Scripcariu, V.; Filipescu, D.; Grigoraş, I. Perioperative Anemia and Transfusion in Colorectal Cancer Patients. Chirurgia 2o19, II4, 234-242. [CrossRef] [PubMed] 
7. Jiang, Z.; Li, Y.; Han, G.; Zhang, J.; Li, Z.; Wang, D.; Liu, Y. Association of serum albumin level with clinicopathologic features and prognosis in colon cancer. Zhonghua Wei Chang Wai Ke Za Zhi 2016, 19, 80-83. [PubMed]

8. Chiang, J.M.; Chang, C.J.; Jiang, S.F.; Yeh, C.Y.; You, J.F.; Hsieh, P.S.; Huang, H.Y. Pre-operative serum albumin level substantially predicts post-operative morbidity and mortality among patients with colorectal cancer who undergo elective colectomy. Eur. J. Cancer Care (Engl.) 2017, 26, eI2403. [CrossRef] [PubMed]

9. Haskins, I.N.; Baginsky, M.; Amdur, R.L.; Agarwal, S. Preoperative hypoalbuminemia is associated with worse outcomes in colon cancer patients. Clin. Nutr. 2or7, 36, 1333-1338. [CrossRef] [PubMed]

Io. Biscond, M.; Guimbaud, R.; Digue, L.; Cirilo-Cassaigne, I.; Bousser, V.; Oum-Sack, E.; Goddard, J.; Bauvin, E.; Delpierre, C.; Grosclaude, P.; et al. How does comorbidity affect colon cancer patients' care trajectory? Results from the French EvaCCoR cohort study. Clin. Res. Hepatol. Gastroenterol. 202I, 45, IOI422. [CrossRef] [PubMed]

II. Roberto, M.; Arrivi, G.; Lo Bianco, F.; Cascinu, S.; Gelsomino, F.; Caputo, F.; Cerma, K.; Ghidini, M.; Ratti, M.; Pizzo, C.; et al. Evaluation of Prognostic Factors for Survival in Transverse Colon Cancer. Cancers 2020, I2, 2457. [CrossRef] [PubMed] 